\title{
End-to-end model for the Polychromatic Laser Guide Star project (ELP-OA)
}

\author{
N. Meilard ${ }^{1, a}$, R. Foy ${ }^{1,2}$, M. Tallon ${ }^{1}$, and É. Thiébaut ${ }^{1}$ \\ 1 Université de Lyon, Lyon, F-69003, FRANCE ; Université Lyon 1, Observatoire de Lyon, 9 av- \\ enue Charles André, Saint-Genis Laval, F-69230, France ; CNRS, UMR 5574, Centre de Recherche \\ Astrophysique de Lyon ; Ecole normale Supérieure de Lyon, Lyon, F-69007, FRANCE \\ 2 Observatoire de Haute-Provence, CNRS USR 2207, F-04870 St Michel l'Observatoire, FRANCE
}

\begin{abstract}
In order to optimize parameters for the ELP-OA project, we have developped an end-to-end model from the laser emission to the computation of the tip-tilt Strehl ratio.

ELP-OA aims at measuring the wavefront tip-tilt from the laser guide star alone, without any natural source. It relies on 2-photon excitation of mesospheric sodium, achieved by two laser chains (at $569 \mathrm{~nm}$ and $589 \mathrm{~nm}$ ) delivering $34 \mathrm{~W}$ average power each. The modeled twin laser beam is launched with a three aperture interferometer that produces an interferometric pattern in the mesosphere after the passage through a Kolmogorov phase screen. The backscattered flux is computed with an optical Bloch equations code [1]. The result of this excitation is an emission at different wavelengths. The wavelengths retained are $330 \mathrm{~nm}, 569 \mathrm{~nm}$ and $589 \mathrm{~nm}$.

An image of this polychromatic LGS (PLGS) is computed, as it will be observed at the $1.52 \mathrm{~m}$ telescope at the Observatoire de Haute-Provence (OHP), through a Kolmogorov phase screen again. Then we simulate the adaptive optics device (adapted from ONERA's BOA AO) at the telescope except for tip-tilt. Air refraction index variations due to atmospheric turbulence create a dispersion between $330 \mathrm{~nm}$ and $569 \mathrm{~nm}$, and the tip-tilt value is derived from this dispersion.

This dispersion between $330 \mathrm{~nm}$ and $569 \mathrm{~nm}$ is measured using a correlation method and a sub-pixel interpolation. The calculation of the relative position between two simultaneous images at $330 \mathrm{~nm}$ and $569 \mathrm{~nm}$ gives a position error, from which the Strehl ratio for tip-tilt is deduced.

At this time, we have got Strehl ratios up to approximatively $35 \%$ at $550 \mathrm{~nm}$, for tilt corrected $r_{0}=10 \mathrm{~cm}$ and a projector baseline of $70 \mathrm{~cm}$.
\end{abstract}

\section{Introduction}

Natural or laser guide stars (LGS) are necessary to allow an adaptive optics system to correct for atmospheric turbulence. Sky coverage is poor with only natural guide stars (NGS). The use of LGS improves the performances, but full sky coverage is still not achieved, especially at visible wavelengths [2].

The main problem is the undetermination of the wavefront tip-tilt. When a LGS is projected, the outgoing tip-tilt prevents the knowledge of its exact position in the mesosphere. So the use of at least one natural guide star is necessary to correct the tip-tilt. Due to the needs of a natural guide star, the sky coverage remains quite poor. This problem is known since many years [3].

ELP-OA is an experience to measure and correct the tip-tilt without the need of a NGS [4], and hence to reach a full sky coverage. This will allow astronomers to observe with $\mathrm{AO}$ in the visible range.

The experience concept is shown in Fig. 1. The PLGS results from the backscattered flux of a 2-photon excitation of mesospheric sodium atoms. From the backscattered flux, measuring the differential tiptilt allow us to derive the global one.

This global tip-tilt is related to the differential one by the following equation:

$$
\theta\left(\lambda_{3}\right)=\frac{n_{\lambda_{3}}-1}{n_{\lambda_{1}}-n_{\lambda_{2}}}\left(\theta\left(\lambda_{1}\right)-\theta\left(\lambda_{2}\right)\right)
$$

\footnotetext{
a e-mail: nicolas.meilard@obs.univ-lyon1.fr
} the original work is properly cited. 


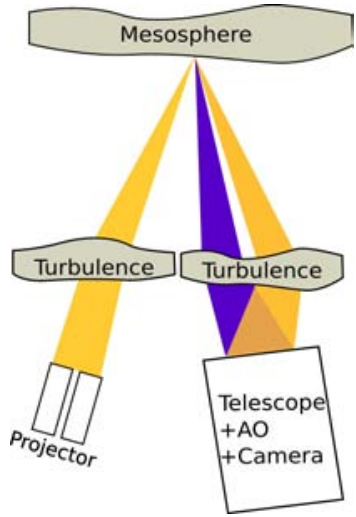

Fig. 1. Concept of ELP-OA experience.

In this relation, $\lambda_{1}$ and $\lambda_{2}$ are the two extracted wavelengths, $\lambda_{3}$ is the correction wavelength and $\theta(\lambda)$ is the tip-tilt at the wavelength $\lambda$. The turbulence tip-tilt is then corrected by the dedicated mirror of the adaptive optics system. To this correction we add telescope vibration compensations.

This experience is being set up at the $1.52 \mathrm{~m}$ telescope of the Observatoire de Haute-Provence (OHP). This article presents the simulation of ELP-OA experience from the laser emission (see Sect. 2) to the data simulation and the tip-tilt correction (see Sect. 3). Results are shown in section 4. In the conclusion we discuss about some projects which absolutely require a PLGS.

\section{Laser star creation}

\subsection{Laser beam projection}

A good correction of the tip-tilt needs a good precision for the measurement of the differential tip-tilt. When decreasing the size of the spot, the increase of this precision is balanced by the loss of photons because of saturation effects. The optimum is obtained with a diameter of the projector close to the diameter of the master telescope. But such a large projector is too expensive and too constraining for the experience.

The solution proposed at Lyon Observatory is to use a three apertures interferometer [5]. With such a device, each aperture is small, and the interferometric pattern in the mesosphere can be as sharp as required. Figure 2 shows such an interferometric pattern.

The advantage of this interferometer is to replace a large single launching telescope by three small

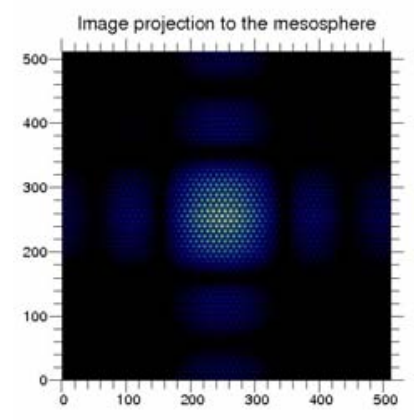

Fig. 2. Example of a mesospheric pattern produced by a three aperture interferometer. 
apertures. But the drawback is not to have a single diffraction-limited pattern in the mesosphere, but a multitude of these patterns: the maximum intensity is drastically reduced.

The three apertures interferometer is subjected to some constrains on the diameter of the apertures and the length of the baselines of the interferometer:

- If the apertures are too large, the mesospheric pattern will have some speckles, and then some random phase shifts of fringes within speckles. They drastically decrease the SNR.

- If the apertures are too small, the mesospheric pattern will be too widely extended and we will not detect enough light from the laser guide star; the extension is also limited by the size of the isoplanatic patch.

- If the interferometer baselines are too small, the fringes will be too large to provide us with a sufficiently accurate measurement of the differential tilt.

- If the interferometer baselines are too long, the mesospheric pattern will be unresolved by the telescope.

This balance results on having diameter of apertures inversely proportionnal to $r_{0}$ and on having interferometer baselines close to the radius of the master telescope. The Sect. 4 presents the results of this balance.

\subsection{Sodium excitation in mesosphere}

The excitation of the mesospheric sodium atoms and the resulting backscattered flux are computed by using the BEACON code [1]. This code uses the optical Bloch equations to compute the number of photons emitted from the mesosphere by fluorescence. The radiative cascade of sodium atom is shown in Fig. 3(a).

BEACON allows the computation of the amount of backscattered flux for different power densities

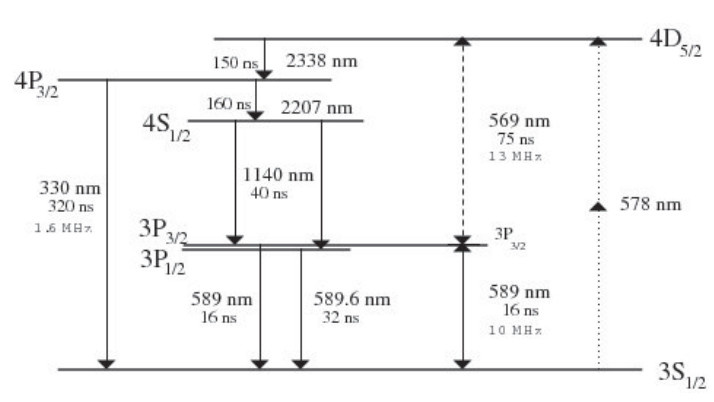

(a) Radiative cascade of sodium.

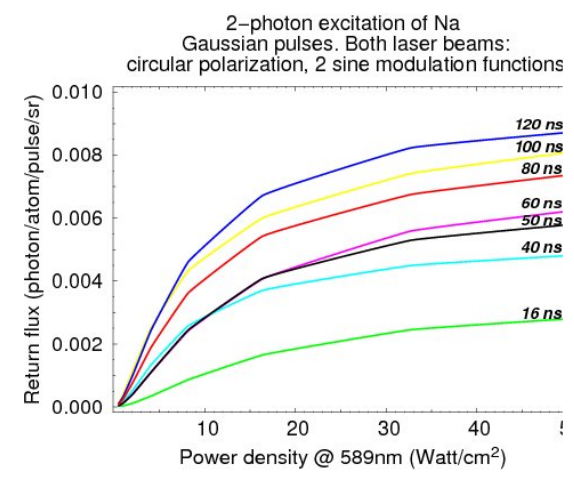

(b) Backscattered flux from $4 D_{5 / 2}$ level.

Fig. 3. Caracteristics of the sodium atoms.

in the mesosphere and different parameters of the laser beams, like the pulsation repetition rate, or the pulse width, as shown in Fig. 3(b) (The two laser beams are considered to have the same intensity distribution in the mesosphere).

\section{Differential tip-tilt measurement}

\subsection{Simulation of data}

The backscattered wavefront goes down to the telescope through a Kolmogorov phase screen and through an AO. The data simulation is made of two steps. The first step is to compute the short exposure 
point spread functions of the telescope at the needed wavelengths, taking into account the turbulence and the partial correction of the adaptive optics. The turbulence is simulated by a Kolmogorov phase screen. The adaptive optics system is simulated by a single deformable mirror conjugated with the entrance pupil. The mirror shape is computed by smoothing the phase aberration: the more smoothing we apply, the less corrected the phase screen is (and the less actuators the deformable mirror would have). Figure 4(b) shows a telescope short exposure point spread function with turbulence, and its correction.

The second step is to compute the LGS images through the telescope. These images are basically the

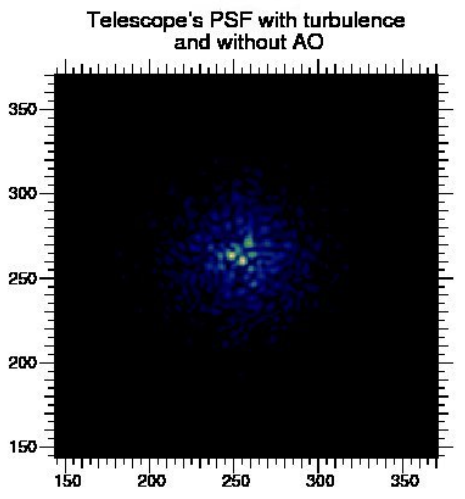

(a) without $\mathrm{AO}$ correction

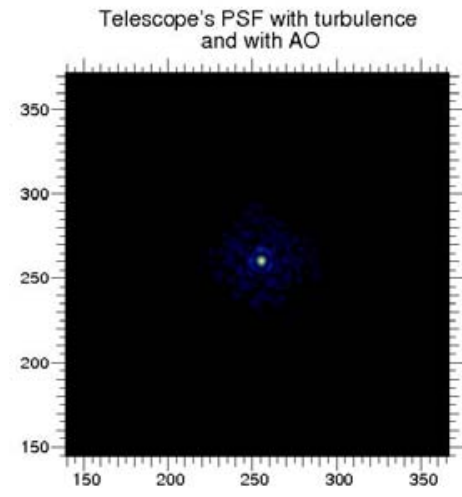

(b) with $\mathrm{AO}$ correction

Fig. 4. "Total" point spread function with turbulence.

LGS backscattered flux, convolved by the telescope point spread functions. Thus, images are blurred by the simulated seeing and then sharpened by the simulated AO, as shown in Fig. 5(a).

In the last step we simulate Poisson noise, both for the photon noise and the multiplication noise of an EMCCD. Poisson realization of the brightness distribution at $569 \mathrm{~nm}$ given by our model is shown in figure 5(b).

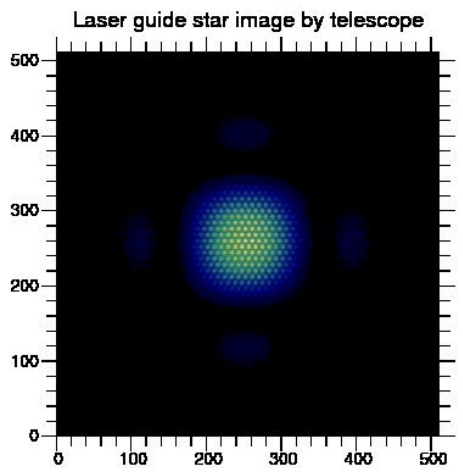

(a) without Poisson noise

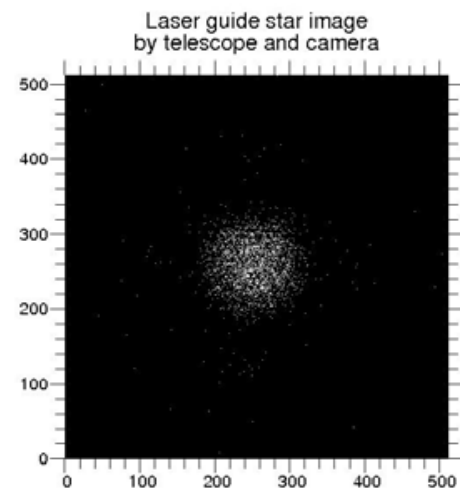

(b) with Poisson noise

Fig. 5. Data simulation : image of the mesospheric pattern image by the telescope after the AO correction. 


\subsection{Matched filter}

Given the two simulated data images at $330 \mathrm{~nm}$ and $569 \mathrm{~nm}$, we have to find their relative positions to get the differential tip-tilt. To that end we use a matched filter [6]. The global tilt is then derived from the differential one as stated before (see Sect. 1).

To use the matched filter, we have to use the data and a model. The data is the LGS images at the AO focus computed by the method described previously (see Sect. 2 and Sect. 3.1). The model is computed in the same way as the data, but without any turbulence nor AO correction nor noise.

The matched filter can be considered with different probability density functions. In our case, we consider the Poisson statistics with the following formula :

$$
\operatorname{Pr}(d \mid \theta)=\prod_{k} \frac{\mathrm{e}^{-m_{k}(\theta)} m_{k}(\theta)^{d_{k}}}{d_{k} !}
$$

with $m_{k}(\theta)=\alpha m\left(x_{k}-t ; \theta\right)$ the mean flux in the $k$-th pixel given the parameters $\theta$ (the brightness factor $\alpha$, the angular position $x_{k}$ of the k-th pixel and the tip-tilt angle $t$ ) and $d_{k}$ the number of photons detected in this pixel.

From this probability, the aim of the method is to find which parameters $t$ (tip-tilt angles to have the data) maximise the following criterion function:

$$
\phi(t)=\left.\frac{\partial(-\log \operatorname{Pr})}{\partial t}\right|_{\alpha=\alpha^{+}}=D \log \frac{M(t)}{D}-\sum_{k} d_{k} m\left(x_{k}-t\right)
$$

where $m\left(x_{k}\right)$ is the model intensity in the k-th pixel, $D$ the data sum on the camera $D=\sum_{k} d_{k}$, and $M(t)$ the model sum on the camera $M(t)=\sum_{\text {detect }} m\left(x_{k}-t\right)$. If the model is not truncated, then $M(t)$ is a constant and the matched filter is simply a convolution (due to the second term). Figure 6 shows the criterion function $\phi(t)$ as a surface ( $t$ is composed from $t_{x}$ and $t_{y}$ ).

The maximum of the criterion gives us the relative translation. From the translations at $330 \mathrm{~nm}$ and at

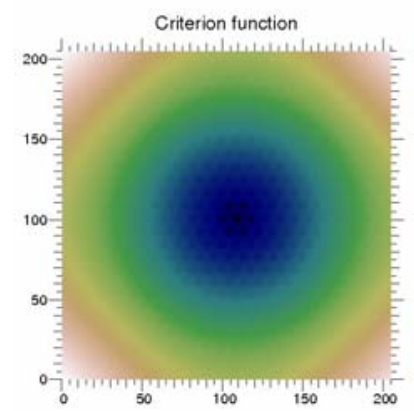

Fig. 6. Criterion function depends on the tip-tilt.

569nm, we derive the tip-tilt and then we correct it.

\section{Results}

The aim of the end-to-end simulation is to help us optimizing some parameters, like the projector baseline, the diameter of projector apertures, the image sampling by the detector, etc.

Figure 7 shows some results that come from our end-to-end model.

Figure 7(a) shows the variation of the tip-tilt Strehl ratio as a function of the projector apertures. The baseline is a constant $(1 \mathrm{~m})$, and the wavelength is $569 \mathrm{~nm}$. The three curves are computed for three 


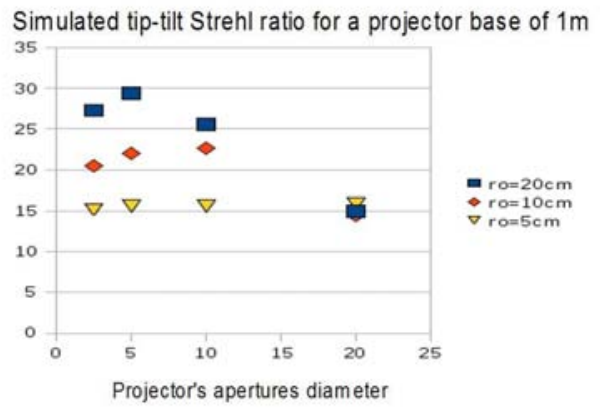

(a) Variation of projector aperture (in meters) diameter with a $1 \mathrm{~m}$ baseline.

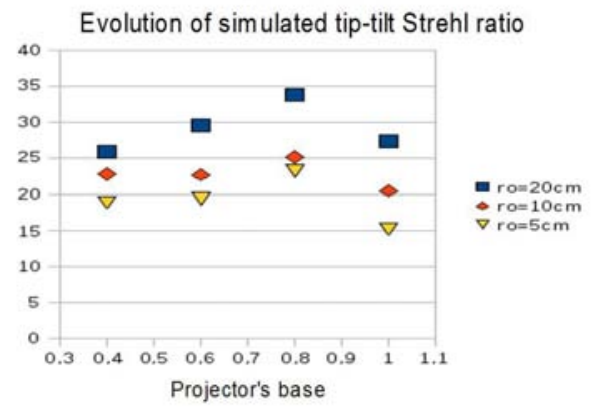

(b) Variation of projector baseline (in meters) with a $25 \mathrm{~mm}$ aperture diameter.

Fig. 7. Strehl ratio at 569nm.

different values of the Fried parameter $(5 \mathrm{~cm}, 10 \mathrm{~cm}$ and $20 \mathrm{~cm})$. The tip-tilt Strehl ratio increases with $r_{0}$. The three curves have a maximum for a diameter of a projector aperture inversely proportionnal of $r_{0}$.

Figure 7(b) shows the variation of the tip-tilt Strehl ratio as a function of the projector baseline. Now, the projector aperture diameter is constant, equal to $25 \mathrm{~mm}$. The three curves correspond to three $r_{0}$ values, the same than figure 7(a). They show a maximum for a $80 \mathrm{~cm}$ baseline, i.e. the telescope radius.

\section{Conclusion}

Our end-to-end model will be completed by geometric effects like the cone effect and anisoplanetism, turbulence coherence time, etc. The end-to-end model will be fitted on experimental measurements obtained at OHP. Then we will be able to predict the performances of a PLGS for 8-10m telescopes and ELTs.

Some applications will be studied with the ELP-OA end-to-end model, like the destruction of space debris. Another application would be the inter-planetary communications e.g. between Earth and Mars.

\section{References}

1. Bellanger V., Courcelle A., Petit A., Computer Physics Communications Volume 162, (2004) 143150

2. Le Louarn M., Foy R., Hubin N. and Tallon M., Monthly Notices of the Royal Astronomical Society Volume 295, (1998) 756-768

3. Rigaut F., Gendron E., Astronomy and Astrophysics Volume 261, (1992) 677-684

4. Foy R., Migus A., Biraben F., Gryuberg G., McCullough P.R., Tallon M., Astronomy and Astrophysics Supplement Series Volume 111, (1995) 569-578

5. Foy R., Blanc P.-É., Fusco T., Laloge A., LeVanSuu A., Meilard N., Perruchot S., Petit A., Tallon M., Thiébaut É., Boër M., This book Abstract 130, (2009)

6. Stewart I. M., Astronomy and Astrophysics Volume 454, (2006) 997-1009 